\title{
Exploring Selective Pressure Trade-Offs for Synthetic Addiction to Extend Metabolite Productive Lifetimes in Yeast
}

Lee, Sang-Woo; Rugbjerg, Peter; Sommer, Morten Otto Alexander

Published in:

ACS Synthetic Biology

Link to article, DOI:

10.1021/acssynbio.1c00240

Publication date:

2021

Document Version

Publisher's PDF, also known as Version of record

Link back to DTU Orbit

Citation (APA):

Lee, S-W., Rugbjerg, P., \& Sommer, M. O. A. (2021). Exploring Selective Pressure Trade-Offs for Synthetic Addiction to Extend Metabolite Productive Lifetimes in Yeast. ACS Synthetic Biology, 10(11), 2842-2849. https://doi.org/10.1021/acssynbio.1c00240

\section{General rights}

Copyright and moral rights for the publications made accessible in the public portal are retained by the authors and/or other copyright owners and it is a condition of accessing publications that users recognise and abide by the legal requirements associated with these rights.

- Users may download and print one copy of any publication from the public portal for the purpose of private study or research.

- You may not further distribute the material or use it for any profit-making activity or commercial gain

- You may freely distribute the URL identifying the publication in the public portal 


\title{
Exploring Selective Pressure Trade-Offs for Synthetic Addiction to Extend Metabolite Productive Lifetimes in Yeast
}

\author{
Sang-Woo Lee, Peter Rugbjerg, and Morten Otto Alexander Sommer*
}

Cite This: ACS Synth. Biol. 2021, 10, 2842-2849

Read Online

ABSTRACT: Engineered microbes often suffer from reduced fitness resulting from metabolic burden and various stresses. The productive lifetime of a bioreactor with engineered microbes is therefore susceptible to the rise of nonproductive mutants with better fitness. Synthetic addiction is emerging as a concept to artificially couple the growth rate of the microbe to production to tackle this problem. However, only a few successful cases of synthetic addiction systems have been reported to date. To understand the limitations and design constraints in long-term cultivations, we designed and studied conditional synthetic addiction circuits in Saccharomyces cerevisiae. This allowed us to

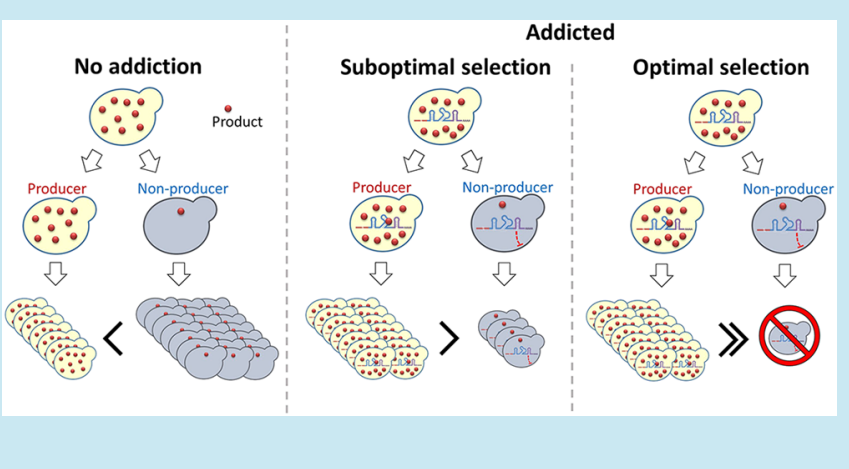
probe a range of selective pressure strengths and identify the optimal balance between circuit stability and production-to-growth coupling. In the optimal balance, the productive lifetime was greatly extended compared with suboptimal circuit tuning. With a too-high or -low pressure, we found that production declines mainly through homologous recombination. These principles of trade-off in the design of synthetic addition systems should lead to the better control of bioprocess performance.

KEYWORDS: industrial biotechnology, synthetic circuit balancing, metabolite production, biosensor, production control, population heterogeneity

\section{INTRODUCTION}

Engineered microorganisms have been extensively used to build a more renewable and sustainable society. However, large-scale operations are required for cost-effective production to compete with chemical processes. The long-term operation of such a bioreactor can suffer from a loss of yield and production due to the natural accumulation of mutations. ${ }^{1-3}$ The stability of metabolite production on an industrial scale has been an important issue because the performance of the bioreactor is directly related to the economic competitiveness of this renewable approach. The cause of the reduced yield in the bioreactor can be explained by single-cell variation owing to stochastic gene expression and mass-transfer heterogeneities. ${ }^{1,4,5}$ However, another important cause of the long-term reduction of yield would be reduced fitness due to metabolic burden, which, in turn, leads to a complete loss of production over time.

For cost-effective operation, microbes are often heavily engineered to produce a maximum titer of metabolites. Metabolic burden commonly arises from certain metabolite depletions, ${ }^{6}$ or toxicities from intermediates and end products ${ }^{7}$ can cause the reduced fitness of engineered host organisms. Spontaneous mutations in these engineered microbes will create nonproductive mutants with better fitness. These nonproducers will take the majority of the population, which leads to a complete loss of production in the bioreactor. ${ }^{3,8}$ Several attempts have been made to solve the genetic instability of engineered microbes. ${ }^{9}$ A common way to achieve this goal is to reduce the mutation rate ${ }^{10,11}$ to slow down the emergence of nonproductive mutants; however, this approach will only delay the reduced yield from arising, and thus a more selective way to extend the productive lifetime would be ideal.

Instead of delaying the occurrence of nonproductive mutants, selecting a producer population with a biosensor coupled to essential genes was proven to be effective to extend the productive lifetime in our recent study. ${ }^{12}$ In this approach, the engineered $E$. coli was addicted to metabolite production to proliferate, thereby selecting the producer population without any conditioned medium. However, the engineering of stable synthetic addiction systems is difficult due to the required circuit tuning, and it is still limited to a few successful cases and the use of a conditioned medium. ${ }^{1,12-15}$ Therefore, to develop better design principles for synthetic product addiction, in this

Received: May 25, 2021

Published: October 26, 2021 

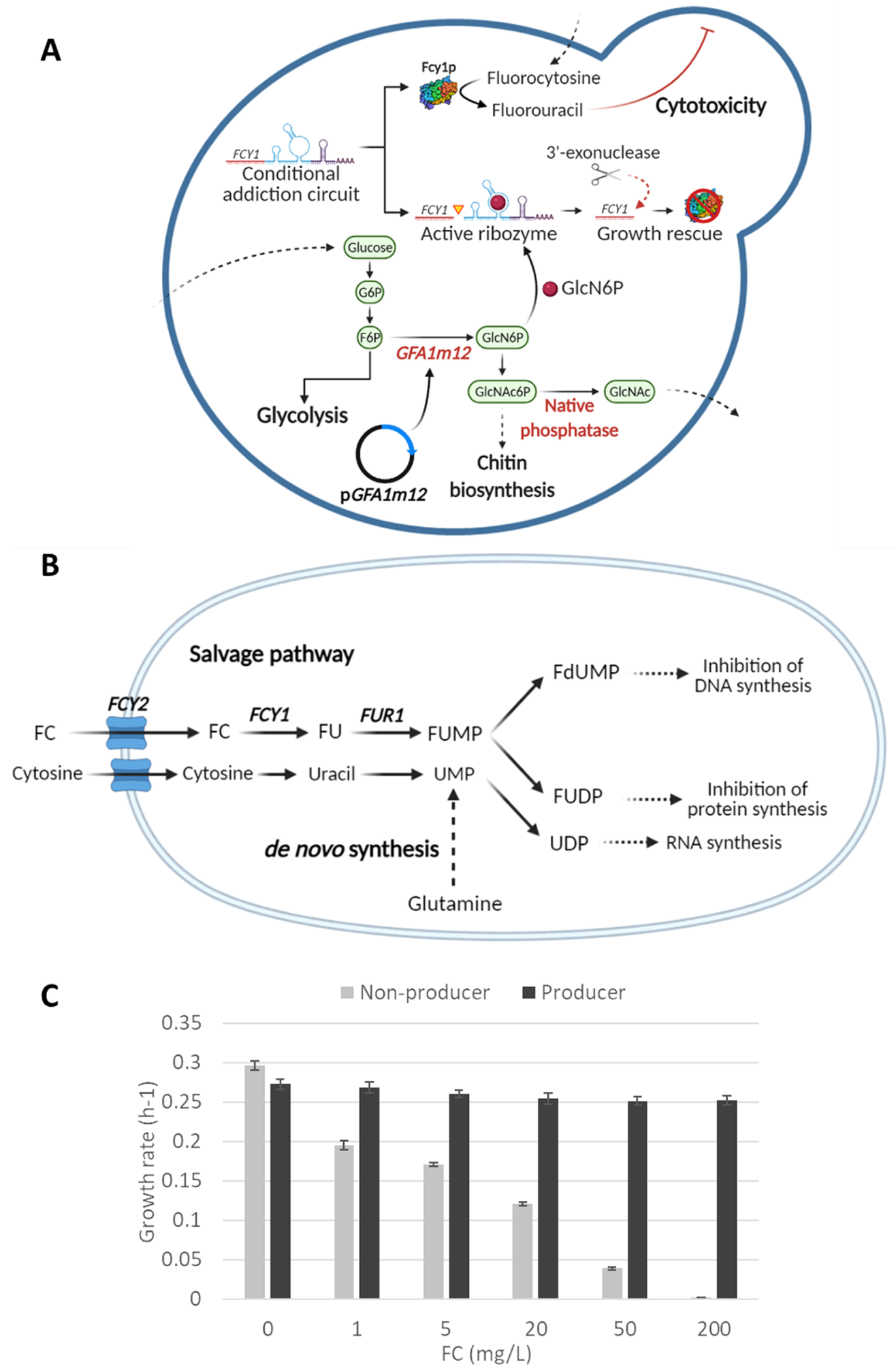

Figure 1. Conditional addiction circuit for yeast S. cerevisiae. (A) Suicide gene FCY1 converts fluorocytosine (FC) to fluorouracil (FU). glmS ribozyme controls the expression of the suicide gene FCY1. A sufficient level of GlcN6P activates glmS ribozyme, which leads to degradation of the FCY1 transcript, thereby restoring the cell growth (synthetic addiction). (B) Mechanism of FC toxicity. FC was imported and converted by three steps (FCY2, FCY1, and FUR1) in the salvage pathway. Disrupting the salvage pathway or activating the de novo synthesis of UMP can show FC resistance. FC: fluorocytosine, FU: fluorouracil, UMP: uridine monophosphate, UDP: uridine diphosphate, FUMP: fluoro-UMP, FdUMP: fluorodeoxy-UMP, FUDP: fluoro-UDP, UPRTase: uracil phosphoribosyltransferase. This figure was inspired by Hope et al., 2004. ${ }^{21}$ (C) Comparison of the specific growth rates of the producer and the nonproducer. Different levels of fluorocytosine (FC) were used to adjust the selective pressure. The producer showed only a minor change in response to the increased selective pressure. The nonproducer, however, showed a significantly reduced growth rate, confirming the usefulness of this biosensor in yeast. Error bars represent standard deviation $(n=3)$. 


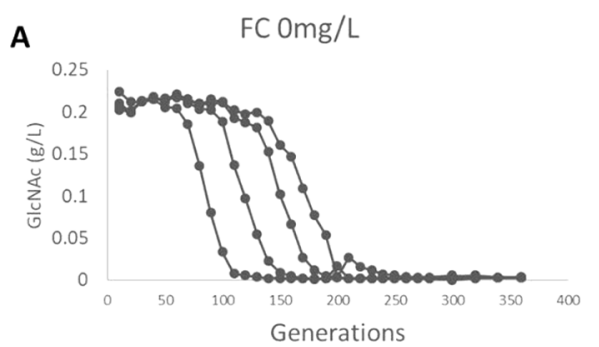

FC $20 \mathrm{mg} / \mathrm{L}$
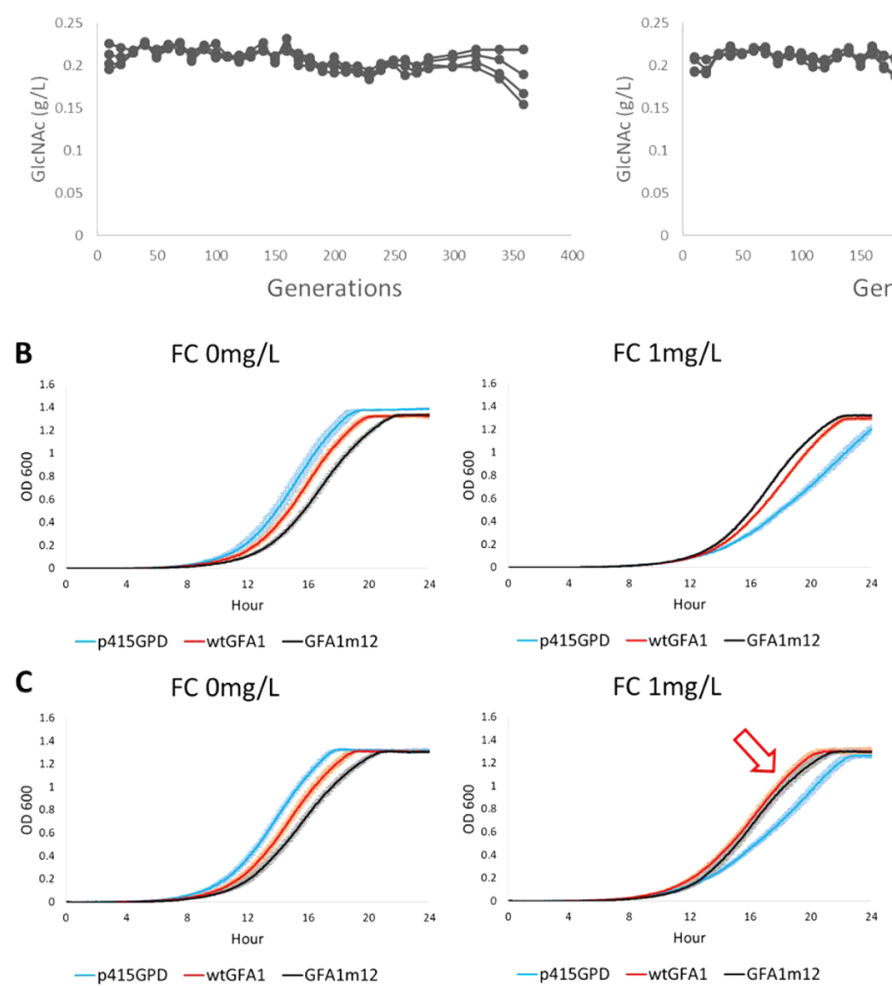

-p415GPD -wTGFA1 -GFA1m12

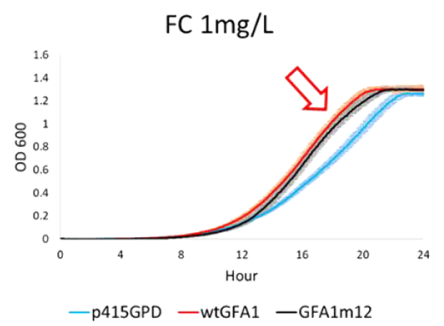

FC $1 \mathrm{mg} / \mathrm{L}$

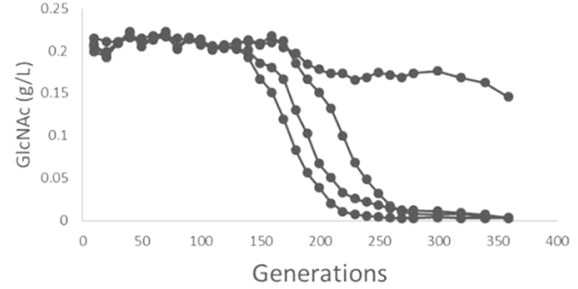

FC 50mg/L
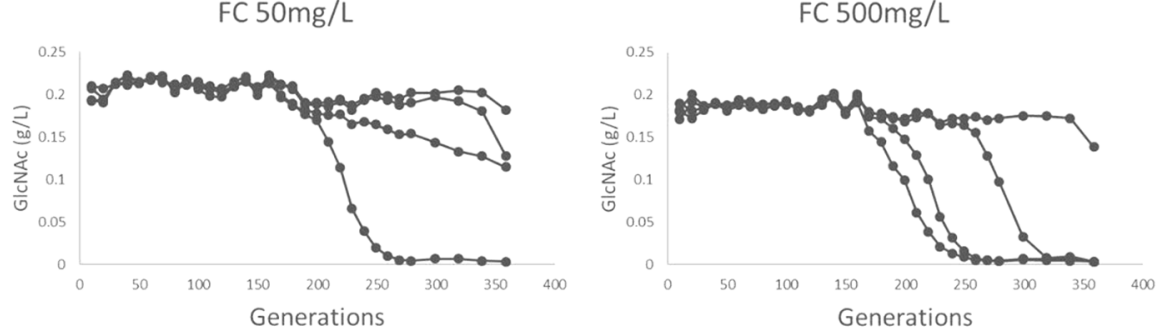

FC 20mg/L
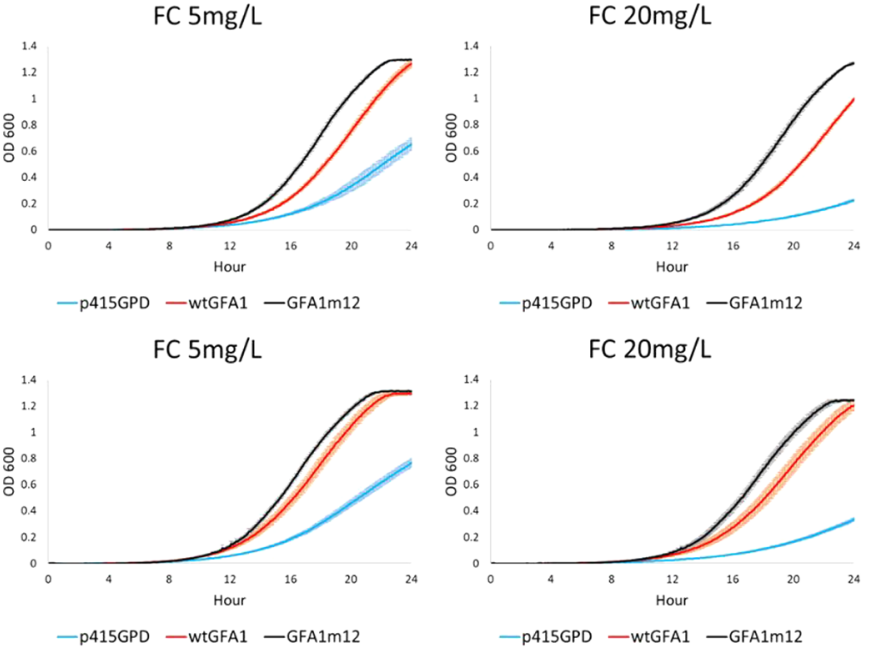

Figure 2. Optimal selection pressure improved production stability. (A) Long-term production was monitored with various selection pressure strengths. Without selective pressure (FC), populations lost production drastically after 100 generations (FC $0 \mathrm{mg} / \mathrm{L}$ ). At low selective pressure $(\mathrm{FC} 1 \mathrm{mg} / \mathrm{L})$ and high selective pressure (FC 50 and $500 \mathrm{mg} / \mathrm{L}$ ), they also lost production after 150 generation. Moderate selective pressure (FC 5 and $20 \mathrm{mg} / \mathrm{L}$ ) maintained production up to 350 generations, proving that optimal selective pressure can significantly extend the productive lifetime. Each line represents a different replicate. (B) Fresh strain with synthetic addiction can select GFA1m12 from wtGFA1 with selective pressure (FC). (C) Strain pregrown in FC $1 \mathrm{mg} / \mathrm{L}$ failed to select GFA1m12 from $w t G F A 1$ only at FC $1 \mathrm{mg} / \mathrm{L}$ (red arrow). Fast adaptation to mild FC stress in yeast seemed to reduce the lag phase, thereby failing selection at the lowest selective pressure (FC $1 \mathrm{mg} / \mathrm{L})$. Error bars represent the standard deviation $(n=4)$.

study, we are exploring its design in Baker's yeast, Saccharomyces cerevisiae, focusing on the tuning of the biosensor-selection interface through a conditional selection gene, FCY1. S. cerevisiae is a commonly used industrial microbe, yet there is limited knowledge of its genetic instability during long-term bioproduction. In S. cerevisiae, a biosensorbased production extension was recently introduced for vanillin $\beta$-glucoside production and monitored on relatively short cultivation scales of 60 generations. ${ }^{14}$ Therefore, it remains unknown if $S$. cerevisiae can sustain production significantly longer, whether such synthetic stabilization circuits themselves collapse, and what are the design principles to sustain addiction over very long-term or even in continuous manufacturing. By understanding the nature of genetic instability, we can also suggest countermeasures to circumvent the emergence of nonproductive mutants, which may greatly improve the economic competence of bioprocess.

\section{RESULTS}

Tunable Circuit for Artificial GIcNAc Addiction in Yeast. Synthetic addiction can couple the growth rate to the production through product-sensitive biosensors and essential genes to stabilize production in long-term cultivation. ${ }^{12} \mathrm{We}$ decided to investigate this strategy further in S. cerevisiae to understand the constraints and design considerations of bioproduction and synthetic stabilization. We hypothesized that an optimized window for selective pressure would provide better production stability and therefore opted for a tunable selection circuit design. In a previous study, we developed a suicide genetic circuit that can isolate efficient enzyme mutants from directed evolution with high-throughput screening in $S$. cerevisiae. ${ }^{16}$ Because this conditional addiction circuit allows a tunable selective pressure through medium supplementation and without additional genetic modification, it was further utilized in this study (Figure 1A). The conditional addiction 
Table 1. Summary of Production Monitoring with Various Selective Pressure Strengths ${ }^{a}$

\begin{tabular}{|c|c|c|c|c|c|c|c|}
\hline & & GlcNAc production & GFA1m12 seq & FC sensitivity & FCY1(circuit) seq & FCY2 seq & FUR1 seq \\
\hline \multirow[t]{4}{*}{ FC $0 \mathrm{mg} / \mathrm{L}$} & rep1 & complete loss & $w t G F A 1$ & intact & & & \\
\hline & rep2 & complete loss & $w t G F A 1$ & intact & & & \\
\hline & rep3 & complete loss & $w t G F A 1$ & intact & & & \\
\hline & rep4 & complete loss & $w t G F A 1$ & intact & R105L & & \\
\hline \multirow[t]{4}{*}{ FC $1 \mathrm{mg} / \mathrm{L}$} & rep 1 & decreasing & intact & intact & & & \\
\hline & rep2 & complete loss & $w t G F A 1$ & intact & & & \\
\hline & rep3 & complete loss & $w t G F A 1$ & resistant & & & \\
\hline & rep4 & complete loss & $w t G F A 1$ & intact & & & \\
\hline \multirow[t]{4}{*}{ FC $5 \mathrm{mg} / \mathrm{L}$} & rep1 & intact & intact & diauxic & & & \\
\hline & rep2 & intact & intact & diauxic & & & \\
\hline & rep3 & intact & intact & partially resistant & & & \\
\hline & rep4 & intact & intact & diauxic & & & \\
\hline \multirow[t]{4}{*}{ FC $20 \mathrm{mg} / \mathrm{L}$} & rep1 & intact & intact & resistant & & T380P & \\
\hline & rep2 & intact & intact & resistant & & T380I & \\
\hline & rep3 & intact & intact & resistant & & & \\
\hline & rep4 & intact & intact & diauxic & & & \\
\hline \multirow[t]{4}{*}{ FC $50 \mathrm{mg} / \mathrm{L}$} & rep1 & complete loss & $w t G F A 1$ & resistant & D92Y & & \\
\hline & rep2 & decreasing & intact & resistant & G96D & & \\
\hline & rep3 & decreasing & intact & resistant/diauxic & frame shift & & \\
\hline & rep4 & decreasing & intact & resistant & & repeat & \\
\hline \multirow[t]{4}{*}{ FC $500 \mathrm{mg} / \mathrm{L}$} & rep1 & complete loss & $w t G F A 1$ & resistant & G14S & & \\
\hline & rep2 & decreasing & intact & resistant & & & \\
\hline & rep3 & complete loss & $w t G F A 1$ & resistant & D11G & & \\
\hline & rep4 & complete loss & MultipleSNPs & resistant & G14C & & \\
\hline
\end{tabular}

${ }^{a}$ Samples at the end of production monitoring were analyzed. The GlcNAc production and the sequencing results for production plasmid (GFA1m12), FCY1, FCY2, and FUR1 are listed.

circuit expresses the suicide gene of $S$. cerevisiae FCY1 in response to the intracellular metabolite level of the target product intermediate glucosamine-6-phosphate (GlcN6P). This feedback is attained through an allosteric self-cleaving ribozyme inserted into a 3 '-untranslated region of FCY1 that reacts with the GlcN6P level and controls the expression of FCY1. The translated Fcylp converts fluorocytosine (FC) to fluorouracil, inducing cytotoxicity. Therefore, different levels of selective pressure can be applied by the FC level in the medium. This conditional addiction circuit is based on a different design than that of our previous report on production control $^{12}$ or another study exploiting auxotrophy/antibiotic resistance in E. coli. ${ }^{1}$ We hypothesized that an FC-dependent genetic circuit could be used to probe which selective pressures will maintain the producer population in long-term cultivation, experimentally simulating the industrial growth duration and the failure modes of the circuit.

To biosynthesize elevated concentrations of GlcNAc (ca. 0.2 $\mathrm{g} / \mathrm{L}$ extracellular), we overexpressed a mutant version of GFA1 (GFA1m12) that was isolated from high-throughput screening using the GlcN6P-responsive biosensor in our previous study. ${ }^{16}$ GFA1 is responsible for a rate-limiting step of cellwall biosynthesis in S. cerevisiae. Because we aimed to monitor the long-term production stability, we overexpressed only GFA $1 m 12$ to make a simplified pathway with a minimal burden rather than having a heavily engineered pathway for $\mathrm{N}$ acetylglucosamine (GlcNAc) production in this study (Figure 1A). We cloned GFA1m12 into the low-copy plasmid with a strong constitutive GPD promoter to make the GlcNAc production plasmid pGFA1m12. Next, we engineered $S$. cerevisiae CEN.PK2-1C to carry both the conditional addiction circuit and the pGFA1m12 and tested for the stability of GlcNAc production in this study.
To evaluate the activity of our conditional addiction circuit, we engineered GlcNAc producer and nonproducer strains and compared them. These engineered strains were grown in different FC concentrations to compare their specific growth rates (Figure 1C). Different concentrations of FC will apply different selective pressures, causing producers and nonproducers to react differently. Nonproducers (strain with empty plasmid) showed an $82 \%$ growth rate reduction when high selective pressure (FC $50 \mathrm{mg} / \mathrm{L}$ ) was applied, whereas producers (strain with GlcNAc production plasmid) showed only a $7.5 \%$ reduction (Figure $1 \mathrm{C}$ at FC $50 \mathrm{mg} / \mathrm{L}$ ), as previously observed in a different strain background. ${ }^{16}$ The growth rate of producers showed an $8 \%$ reduction compared with that of nonproducers when no selective pressure was applied (Figure 1C at FC $0 \mathrm{mg} / \mathrm{L}$ ). This fitness cost of production was caused by expressing GFA1m12, and we therefore hypothesized that spontaneous mutations on the production plasmid will restore the growth rate. Therefore, the overall production will decrease over time in long-term cultivation without any selective pressure.

To validate our conditional addiction circuit, we monitored engineered strains with the GlcNAc production plasmid pGFA1m12 in long-term cultivation to compare with and without selective pressure as a preliminary test. $0.1 \%$ of cultivated samples with the engineered strain were transferred to fresh medium every $48 \mathrm{~h}$, and the GlcNAc level was monitored in each transfer. As we expected, populations without selective pressure (without FC) lost their production drastically after 100 generations (around 10 transfers) (Supplementary Figure 1). On the contrary, populations with selective pressure (with FC $25 \mathrm{mg} / \mathrm{L}$ ) kept their production until 250 generations, proving the usefulness of the production control of our conditional addiction circuit. We hypothesized 
that proper selective pressure can further extend the productive lifetime; therefore, we tried to exploit the tunability of our conditional addiction circuit in the next step.

Production Monitoring Showed Moderate Selective Pressure Is Favorable. We hypothesized that optimal selective pressure should be important to extend the productive lifetime when using synthetic addiction systems. If selective pressure is too weak, then we anticipated that nonproducers will not be eliminated properly, and over time, they will eventually dominate the population. At the same time, we speculated that strong pressure will cause an additional burden that could compromise the integrity of the selection system. To address this question, we decided to monitor our engineered strain with various ranges of selective pressure, expecting that an unknown, optimal point could extend the productive lifetime significantly better.

Serial transfer and GlcNAc level detection were carried out as in a preliminary test to experimentally simulate long-term cultivation using different selection pressures. As we expected, control samples without any selective pressure started to lose their production around 100 generations, similar to the previous test (Figure 2A, FC $0 \mathrm{mg} / \mathrm{L}$ ). Interestingly, samples with the lowest and highest selective pressures started to lose their production around 150 generations following the control sample (FC 1 and $500 \mathrm{mg} / \mathrm{L}$, respectively). Samples with relatively high selective pressure (FC $50 \mathrm{mg} / \mathrm{L}$ ) also showed poor stability, whereas moderate selective pressure (FC 5 and $20 \mathrm{mg} / \mathrm{L}$ ) performed the best. Indeed, moderate selective pressure could extend a productive lifetime of our strain from 100 generations to at least 350 generations, proving that a more cost-effective operation for the industrial scale is possible with synthetic selection.

Declining Production and Circuit Function Are Genetically Explained by Homologous Recombination and FC Resistance Development. We observed that moderate selective pressure greatly the extended productive lifetime, whereas populations experiencing low or high selective pressure lost their GlcNAc production much earlier in our production monitoring (Figure 2A). To verify the underlying mechanism of their behavior, we further collected and analyzed samples after the last transfer. Their GlcNAc production and sequencing results for the production plasmid (GFA1m12), FCY1 (addiction circuit), FCY2, and FUR1 are summarized in Table 1. In all examples of lost GlcNAc production, we found mutations on the GFA1m12-encoding gene of the production plasmid. Developed FC resistance could be explained by mutations on the FCY1, FCY2, and FUR1 loci from the genome.

Interestingly, the common error mode on the production plasmid was the gene conversion of GFA1m12 to wild-type GFA1 (wtGFA1) by homologous recombination (Table 1). Because GFA1m12 requires a 4 base pair (bp) substitution to revert to $w t G F A 1$, it is unlikely that this evolution is caused by a spontaneous mutation. All samples that showed a complete loss of GlcNAc production had wild-type GFA1 instead of GFA $1 \mathrm{~m} 12$ on their production plasmid, except for FC $500 \mathrm{mg} /$ $\mathrm{L}$ rep4, which had multiple mutations. GFA1m12 was isolated from directed evolution with the error-prone polymerase chain reaction (PCR) and has four amino acid substitutions (V12L, Q96H, Q157R, and E343V) compared with the wild-type GFA1 sequence. ${ }^{16}$ These mutations seemingly eliminated the allosteric inhibition of Gfalp by uridine diphosphate $N$ acetylglucosamine (UDP-GlcNAc), an end product of the chitin biosynthetic pathway. ${ }^{16}$ Therefore, we can expect that reversion to wild-type GFA1 will significantly reduce the GlcNAc production.

Wild-type GFA1 on the genome of CEN.PK 2-1C was not deleted in this study because it is an essential gene for chitin and cell-wall biosynthesis. However, strong homologous recombination activity in yeast reverted GFA $1 \mathrm{~m} 12$ to wildtype GFA1 in long-term cultivation, which leads to reduced fitness costs caused by GlcNAc production. Indeed, we observed that expressing wild-type GFA1 on the same plasmid backbone had a better growth rate than GFA1m12, although it was lower than that of the empty plasmid (Figure 2B, FC 0 $\mathrm{mg} / \mathrm{L})$. The strain with a plasmid expressing wild-type GFA1 did not secrete a detectable amount of GlcNAc; however, this strain was able to grow slightly faster than the GFA1m12 strain when the strain was pregrown at FC $1 \mathrm{mg} / \mathrm{L}$ once and tested (Figure 2C, FC $1 \mathrm{mg} / \mathrm{L}$ ). This explains why the producer population was not properly selected at the lowest selective pressure (Figure 2A and Table 1, FC $1 \mathrm{mg} / \mathrm{L}$ ). Fresh strains with a conditional addiction circuit can select GFA1m12 over wild-type GFA1, even at FC $1 \mathrm{mg} / \mathrm{L}$, when they encounter selective pressure for the first time (Figure 2B, FC $1 \mathrm{mg} / \mathrm{L}$ ); however, yeast strains seemed to adapt quite quickly to mild FC stress, ${ }^{17}$ and this leads to a slightly reduced lag time, resulting in the failure to select the producer population at the lowest selective pressure (Figure 2B,C at FC $1 \mathrm{mg} / \mathrm{L}$ ).

Too-High Selective Pressure Selects for Resistance Development and Lost Addiction Function. Another interesting observation from our analysis is that relatively high selective pressure developed strong FC resistance and eventually disabled the genetic circuit. FC is a commonly used antifungal drug, and the molecular mechanism to confer resistance is well studied in the pathogenic yeast background. ${ }^{18-21}$ This antifungal drug can inhibit DNA replication, transcription, and protein synthesis. ${ }^{22,23}$ There are two major routes to confer resistance. One is by disrupting the pyrimidine salvage pathway (FCY1, FCY2, and FUR1), and the other is by increasing the de novo pyrimidine synthesis (Figure $1 \mathrm{~B}$ ). ${ }^{20}$

At higher selective pressure (FC 50 and $500 \mathrm{mg} / \mathrm{L}$ ) in our monitoring, populations tend to disrupt the pyrimidine salvage pathway to survive severe stress from FC toxicity (Table 1). Evolved populations under this condition seemed to disrupt FCY1, FCY2, and FUR1 directly to confer strong FC resistance. Indeed, populations under higher selective pressure (FC 50 and $500 \mathrm{mg} / \mathrm{L}$ ) almost completely lost FC sensitivity whether or not they had intact GFA1m12 (Table 1 and Supplementary Figure 2). A disruption in the salvage pathway was known to show high FC resistance in S. cerevisiae; ${ }^{24-27}$ therefore, cells under higher FC concentration lose FC sensitivity and disable the genetic circuit to survive in high selective pressure, resulting in a loss of production in long-term cultivation. We confirmed this theory by observing populations in decreasing production at the highest selective pressure $(\mathrm{FC} 500 \mathrm{mg} / \mathrm{L}$ rep3 at \#22 transfer). Nonproducers were the dominating producers because the conditional addiction circuit was disabled under this condition (Supplementary Figure 3). On the contrary, populations at lower selective pressure (FC 5 $\mathrm{mg} / \mathrm{L}$ and lower) mostly maintained an intact FC sensitivity and therefore showed different responses when they had intact GFA1m12 or wtGFA1 (Table 1 and Supplementary Figure 2). Interestingly, populations showed a diauxic growth pattern mostly at moderate selective pressure (FC $5 \mathrm{mg} / \mathrm{L}$ ), suggesting that populations adapted to mild FC stress without disrupting 
the pyrimidine salvage pathway (Table 1 and Supplementary Figure 2).

Homologous Recombination Was a Major Mechanism of Production Decline. Because 10 out of 11 samples that completely lost production had converted GFA1m12 to $w t G F A 1$ on their production plasmid (Table 1), the major mechanism of production decline in our system is likely to be homologous recombination. To further characterize the mechanisms of decline, we investigated single colonies from streaks of several samples featuring decreasing production but not a complete loss (Supplementary Figure 4). Replicate 1 from FC $1 \mathrm{mg} / \mathrm{L}$ and replicate 2 from FC $50 \mathrm{mg} / \mathrm{L}$ consisted of a mixed population with producer cells (intact GFA1m12) and escaper cells ( $w t G F A 1$ ). Replicate 3 from FC $50 \mathrm{mg} / \mathrm{L}$ and replicate 2 from FC $500 \mathrm{mg} / \mathrm{L}$ showed only a minor decrease at the end of the production monitoring. This explains why a production decline was not captured from eight selected colonies. It also suggests that there will be a minor population showing a complete loss of production by homologous recombination.

Interestingly, replicate 4 from FC $50 \mathrm{mg} / \mathrm{L}$ showed a linear decrease in production monitoring, and the major population showed a $\sim 20 \%$ reduced production; however, the GPD promoter and GFA1m12 ORF sequence were intact (Supplementary Figure 4), suggesting that mutations downstream of the GlcNAc pathway, outside of the production plasmid, caused the slightly reduced production. Further studies should include whole-genome sequencing, but we speculate possible mutation targets to include the gene encoding Gnalp converting GlcN6P to GlcNAc6P or native phosphatase (Figure 1A).

\section{DISCUSSION}

Microorganisms used in bioreactors are heavily engineered to focus on the higher yield and production of the metabolite; ${ }^{28}$ however, once they lose their production by spontaneous mutations, nonproductive mutants will dominate the entire population in the reactor due to the better fitness. Therefore, the original population producing a high level of metabolite will be outgrown by these nonproducers. This problem leads to reduced production and impaired overall performance, thereby hampering the economic operation of sustainable alternatives against chemical processes.

In this study, our genetic circuit selected high-performing producers against nonproducers (Figure 1C). One advantage of this circuit is that it can apply different selective pressure by modulating the FC concentration in the medium without introducing genetic modifications. With tunable selective pressure control, we showed that optimal selective pressure can significantly extend the productive lifetime (Figure 2A).

In our observation, synthetic addiction could not enrich producers (strain with intact GFA1m12) from escapers (strain with reverted $w t G F A 1$ ) at the lowest selective pressure (Figure 2C, FC $1 \mathrm{mg} / \mathrm{L}$ ). Although this condition can select producers (strain with GFA1m12) from nonproducers (strain with empty plasmid), evolved populations mostly have reverted wtGFA1 due to the strong homologous recombination activity in yeast. Our previous study in engineered Escherichia coli showed that the common error mode was disruption by mobile elements. $^{8,12}$ These results imply that one should consider a common error mode of engineered microbes to find an optimal biosensor design. Strong recombination activity in yeast can be applied for various purposes ${ }^{29-32}$ but can also cause instability in the engineered strain. ${ }^{14}$ We expect that deleting the potential homology region (GFA1 locus on the genome in our case) will further improve the production stability; therefore, understanding the common error mode will be critical to operating long-term cultivation with engineered hosts.

At extreme selective pressure (FC $500 \mathrm{mg} / \mathrm{L}$ ), populations maintained production slightly longer than at low selective pressure (Figure 2A); however, we observed that evolved populations under this condition tend to develop/evolve FC resistance before the production plasmid is damaged (Supplementary Figure 3). A loss of FC sensitivity caused by the disrupted pyrimidine salvage pathway leads to the disabling of our conditional synthetic addiction. Therefore, it could not select the producer population and was susceptible to the rise of nonproducers, which explains its poor performance at maintaining production stability. In contrast, a moderate selective pressure (FC $5 \mathrm{mg} / \mathrm{L}$ ) maintained both the FC sensitivity and the GlcNAc production. The GlcNAc productive lifetime was greatly extended to 350 generations (Figure 2A). Samples from FC $20 \mathrm{mg} / \mathrm{L}$ developed FC resistance (Table 1 and Supplementary Figure 2), although GlcNAc production was maintained, suggesting that the addiction circuit cannot select the producer population any longer, as we can observe a slight decline at the end of monitoring (Figure 2A, FC $20 \mathrm{mg} / \mathrm{L}$ ). Therefore, we can conclude that the optimal selective pressure for this addiction circuit is around FC $5 \mathrm{mg} / \mathrm{L}$. These results confirmed that a proper balance in selective pressure is important for the longterm cultivation of engineered microbes.

Although our genetic circuit is useful for stable long-term production, it requires additional fluorocytosine in the medium, which could cause additional cost and downstream processing. Therefore, it is preferable to replace fluorocytosine, an environmentally problematic antifungal drug, for large-scale industrial operations. A modified genetic circuit that does not require additional cost can be used instead. Several other candidates to induce cytotoxicity can be tested to replace the suicide gene FCY1, which requires fluorocytosine. Genes inducing cytotoxicities such as GIN $11 M 86^{33}$ or $P K A 3^{34}$ can be tested in the future to improve our strategy. In this case, it will require fine-tuning of the expression level to optimize the selective pressure properly. For continuous fermentation processes, these approaches for studying genetic heterogeneity could be even more important and should be conducted in settings that more closely mimic the continuous fermentation process.

Synthetic addiction can enrich the producer population against the nonproducers, but their performance depends on their context in long-term cultivation. ${ }^{12,14}$ Transcription-factorbased biosensors controlled the essential gene expression in these examples. On the basis of our observation from conditional synthetic addiction in this study, fine-tuning essential gene expression levels can potentially reduce the occurrence of escaper mutants resistant to selective pressure and further improve the productive lifetime. Applying various translational strengths (including ribosome binding site (RBS) optimization) for essential gene expression, for example, can help us to find an optimal point for synthetic addiction in the future. 


\section{METHODS}

Strain and Plasmid Construction. Similar to our previous study, ${ }^{16}$ a conditional addiction circuit and production plasmid were cloned and introduced to the Saccharomyces cerevisiae CEN.PK2-1C strain to make the $\mathrm{N}$ acetylglucosamine (GlcNAc) producer strain in this study. In brief, the integration fragment consisting of the $g l m S$ ribozyme and the URA3 cassette synthesized as a gBlock (IDT, Coralville, IA) was PCR-amplified using FGU insertion F/R primers and inserted into $3^{\prime}-\mathrm{UTR}$ of the FCY1 locus in the CEN.PK2-1C strain by the LiAc transformation method to make a conditional addiction circuit strain SAY. GFA1m12 (synthesized by IDT as a gBlock) from the previous study ${ }^{16}$ and wild-type GFA1 were cloned into the p415GPD plasmid ${ }^{35}$ by USER cloning ${ }^{36}$ using GFA1m 12 USER F/R and p415GPD $\mathrm{BB} F / \mathrm{R}$ primers to make plasmids pGFA1m12 and pGFA1, respectively. p415GPD, pGFA1, and pGFA1m12 plasmids were transformed into the SAY strain to construct a nonproducer (with p415GPD), escaper (with pGFA1), and producer (with pGFA1m12) strain. Strains, plasmids, and primers used in this study are listed in Supplementary Table 1.

Cultivation Conditions for Production Monitoring. The strains were cultivated on a synthetic defined minimal medium containing $20 \mathrm{~g} / \mathrm{L}$ glucose, $6.7 \mathrm{~g} / \mathrm{L}$ yeast nitrogen base without amino acids, and $1.6 \mathrm{~g} / \mathrm{L}$ yeast synthetic drop-out medium supplements without leucine (SD-Leu). For long-term production monitoring, strains were cultivated in a 24-deepwell plate with $1 \mathrm{~mL}$ of the medium. $1 \mu \mathrm{L}$ of the previous culture was transferred to a new plate containing fresh medium every 2 days ( $0.1 \%$ transfer). We applied various selective pressures with different FC concentrations $(0,1,5,20,50$, and $500 \mathrm{mg} / \mathrm{L})$, and four replicates were monitored for each concentration. After each transfer, cultures were centrifuged, and cell pellets were freeze-stored. The supernatant was also stored to determine the extracellular GlcNAc level.

Determination of GIcNAc Titer by High-Performance Liquid Chromatography. The extracellular GlcNAc level was determined by high-performance liquid chromatography (HPLC), similar to a previous study. ${ }^{37}$ In brief, the culture medium was centrifugated, and the supernatant was analyzed by HPLC. The GlcNAc peak was detected at $205 \mathrm{~nm}$ wavelength by a UV detector (Ultimate 3000 HPLC system) at $12 \mathrm{~min}$ in the Aminex HPX-87H column $(300 \times 7.8 \mathrm{~mm})$ using $5 \mathrm{mM} \mathrm{H}_{2} \mathrm{SO}_{4}$ as an eluent at $60{ }^{\circ} \mathrm{C}$.

Sequencing Analysis of Plasmid and Salvage Pathway Genes from Production Monitoring. We extracted the genomic DNA and plasmid from samples after \#36 transfer to verify the sequence of the production plasmid and salvage pathway genes. The freeze-stored cell from the \#36 transfer was cultivated again in SD-Leu medium, and cells were extracted for genomic DNA ${ }^{38}$ and plasmid DNA (Zymoprep Yeast Plasmid Miniprep II). To check FCY1 and the addiction circuit sequence, we used primer FGU seq F/R for the PCR amplification from the extracted genomic DNA and the subsequent Sanger sequencing. Similarly, FCY2 seq F/R and FUR1 seq F/R were used for the FCY1 and FUR1 sequences, respectively. The GPD promoter and GFA1m12 sequence on the production plasmid were PCR-amplified and verified by Sanger sequencing with the GPD promoter F and GFA1m12 USER $\mathrm{F} / \mathrm{R}$ primers from the extracted plasmid DNA. Sequencing data were aligned to check the mutation sites compared with the wild-type sequence.
Growth Monitoring of Engineered Strains with a Microtiter Plate Reader. We monitored the growth profile of the engineered strains with a microtiter plate reader (Synergy H1) similar to our previous study. ${ }^{12}$ In brief, freezestored strains were streaked on the SD-Leu plate. A single colony from each strain was cultured and monitored with double-orbital shaking $(550 \mathrm{rpm})$ at $30{ }^{\circ} \mathrm{C}$ in 48 -well plates (Corning 3548) containing $500 \mu \mathrm{L}$ of SD-Leu medium. The $\mathrm{OD}_{600}$ was measured every $5 \mathrm{~min}$ with different $\mathrm{FC}$ concentrations in the medium to compare their growth profiles.

\section{ASSOCIATED CONTENT}

\section{Supporting Information}

The Supporting Information is available free of charge at https://pubs.acs.org/doi/10.1021/acssynbio.1c00240.

Supplementary Table 1. Strain, plasmid, and primers. Supplementary Figure 1. Preliminary production monitoring with an addiction system. Supplementary Figure 2. FC sensitivity of different samples after production monitoring. Supplementary Figure 3. Confirmation that FC resistance was developed before production decline in the highest selective pressure. Supplementary Figure 4. Samples with reduced production were also likely to be caused by homologous recombination (PDF)

\section{AUTHOR INFORMATION}

\section{Corresponding Author}

Morten Otto Alexander Sommer - Novo Nordisk

Foundation Center for Biosustainability, Technical University of Denmark, 2800 Kongens Lyngby, Denmark; 자이.org/ 0000-0003-4005-5674; Email: msom@bio.dtu.dk

\section{Authors}

Sang-Woo Lee - Novo Nordisk Foundation Center for Biosustainability, Technical University of Denmark, 2800 Kongens Lyngby, Denmark

Peter Rugbjerg - Novo Nordisk Foundation Center for Biosustainability, Technical University of Denmark, 2800 Kongens Lyngby, Denmark; Enduro Genetics ApS, 2200 Copenhagen, Denmark

Complete contact information is available at: https://pubs.acs.org/10.1021/acssynbio.1c00240

\section{Author Contributions}

S.-W.L., P.R., and M.O.A.S. designed the research. S.-W.L. performed the experiment. S.-W.L., P.R., and M.O.A.S. analyzed the data. S.-W.L., P.R., and M.O.A.S. wrote the manuscript.

Funding

This research was supported by the Novo Nordisk Foundation under NNF grant number NNF20CC0035580.

\section{Notes}

The authors declare the following competing financial interest(s): P.R. has a financial interest in Enduro Genetics ApS, which holds proprietary synthetic addiction technology.

\section{ABBREVIATIONS}

GlcNAc, N-acetylglucosamine; GlcN6P, glucosamine-6-phosphate; UDP-GlcNAc, uridine diphosphate $\mathrm{N}$-acetylglucosamine; FC, fluorocytosine; $w t G F A 1$, wild-type GFA1; GFA1m12, efficient mutant of GFA1 


\section{REFERENCES}

(1) Xiao, Y.; Bowen, C. H.; Liu, D.; Zhang, F. Exploiting nongenetic cell-to-cell variation for enhanced biosynthesis. Nat. Chem. Biol. 2016, 12, 339-344.

(2) Rugbjerg, P.; Sommer, M. O. A. Overcoming genetic heterogeneity in industrial fermentations. Nat. Biotechnol. 2019, 37, 869-876.

(3) Rugbjerg, P.; Myling-Petersen, N.; Porse, A.; Sarup-Lytzen, K.; Sommer, M. O. A. Diverse genetic error modes constrain large-scale bio-based production. Nat. Commun. 2018, 9, 787.

(4) Heins, A.-L.; Lencastre Fernandes, R.; Gernaey, K. V.; Lantz, A. E. Experimental and in silico investigation of population heterogeneity in continuous Sachharomyces cerevisiae scale-down fermentation in a two-compartment setup. J. Chem. Technol. Biotechnol. 2015, 90, 324340.

(5) Mustafi, N.; Grünberger, A.; Kohlheyer, D.; Bott, M.; Frunzke, J. The development and application of a single-cell biosensor for the detection of 1-methionine and branched-chain amino acids. Metab. Eng. 2012, 14, 449-457.

(6) Glick, B. R. Metabolic load and heterologous gene expression. Biotechnol. Adv. 1995, 13, 247-261.

(7) Pitera, D. J.; Paddon, C. J.; Newman, J. D.; Keasling, J. D. Balancing a heterologous mevalonate pathway for improved isoprenoid production in Escherichia coli. Metab. Eng. 2007, 9 (2), 193-207.

(8) Rugbjerg, P.; Dyerberg, A. S. B.; Quainoo, S.; Munck, C.; Sommer, M. O. A. Short and long-read ultra-deep sequencing profiles emerging heterogeneity across five platform Escherichia coli strains. Metab. Eng. 2021, 65, 197.

(9) Bull, J. J.; Barrick, J. E. Arresting Evolution. Trends Genet. 2017, 33, 910-920.

(10) Sleight, S. C.; Bartley, B. A.; Lieviant, J. A.; Sauro, H. M. Designing and engineering evolutionary robust genetic circuits. J. Biol. Eng. 2010, 4, 12.

(11) Suarez, G. A.; Renda, B. A.; Dasgupta, A.; Barrick, J. E. Reduced Mutation Rate and Increased Transformability of Transposon-Free Acinetobacter baylyi ADP1-ISx. Appl. Environ. Microbiol. 2017, 83 (17), e01025-17.

(12) Rugbjerg, P.; Sarup-Lytzen, K.; Nagy, M.; Sommer, M. O. A. Synthetic addiction extends the productive life time of engineered Escherichia coli populations. Proc. Natl. Acad. Sci. U. S. A. 2018, 115, 2347-2352.

(13) Lv, Y.; Gu, Y.; Xu, J.; Zhou, J.; Xu, P. Coupling metabolic addiction with negative autoregulation to improve strain stability and pathway yield. Metab. Eng. 2020, 61, 79-88.

(14) D’Ambrosio, V.; Dore, E.; Di Blasi, R.; van den Broek, M.; Sudarsan, S.; Horst, J. t.; Ambri, F.; Sommer, M. O. A.; Rugbjerg, P.; Keasling, J. D.; Mans, R.; Jensen, M. K. Regulatory control circuits for stabilizing long-term anabolic product formation in yeast. Metab. Eng. 2020, 61, 369-380.

(15) Wang, X.; Cabales, A.; Li, Z.; Zhang, H. Biosensor-assisted high performing cell selection using an E. coli toxin/antitoxin system. Biochem. Eng. J. 2019, 144, 110-118.

(16) Lee, S. W.; Oh, M. K. A synthetic suicide riboswitch for the high-throughput screening of metabolite production in Saccharomyces cerevisiae. Metab. Eng. 2015, 28, 143-150.

(17) Costa, C.; Ponte, A.; Pais, P.; Santos, R.; Cavalheiro, M.; Yaguchi, T.; Chibana, H.; Teixeira, M. C. New Mechanisms of Flucytosine Resistance in C. glabrata Unveiled by a Chemogenomics Analysis in S. cerevisiae. PLoS One 2015, 10, No. e0135110.

(18) Francis, P.; Walsh, T. J. Evolving role of flucytosine in immunocompromised patients: new insights into safety, pharmacokinetics, and antifungal therapy. Clin. Infect. Dis. 1992, 15, 10031018.

(19) Polak, A. 5-Fluorocytosine-current status with special references to mode of action and drug resistance. Contrib. Microbiol. Immunol. 1977, 4, 158-167.
(20) Vermes, A.; Guchelaar, H. J.; Dankert, J. Flucytosine: a review of its pharmacology, clinical indications, pharmacokinetics, toxicity and drug interactions. J. Antimicrob. Chemother. 2000, 46, 171-179.

(21) Hope, W. W.; Tabernero, L.; Denning, D. W.; Anderson, M. J. Molecular mechanisms of primary resistance to flucytosine in Candida albicans. Antimicrob. Agents Chemother. 2004, 48, 4377-4386.

(22) Espinel-Ingroff, A. Mechanisms of resistance to antifungal agents: yeasts and filamentous fungi. Revista iberoamericana de micologia 2008, 25, 101-106.

(23) Ghannoum, M. A.; Rice, L. B. Antifungal agents: mode of action, mechanisms of resistance, and correlation of these mechanisms with bacterial resistance. Clin. Microbiol. Rev. 1999, 12, 501-517.

(24) Kurtz, J. E.; Exinger, F.; Erbs, P.; Jund, R. New insights into the pyrimidine salvage pathway of Saccharomyces cerevisiae: requirement of six genes for cytidine metabolism. Curr. Genet. 1999, 36, 130-136.

(25) Erbs, P.; Exinger, F.; Jund, R. Characterization of the Saccharomyces cerevisiae FCY1 gene encoding cytosine deaminase and its homologue FCA1 of Candida albicans. Curr. Genet. 1997, 31, $1-6$.

(26) Guetsova, M. L.; Lecoq, K.; Daignan-Fornier, B. The isolation and characterization of Saccharomyces cerevisiae mutants that constitutively express purine biosynthetic genes. Genetics 1997, 147, 383-397.

(27) Kern, L.; de Montigny, J.; Lacroute, F.; Jund, R. Regulation of the pyrimidine salvage pathway by the FUR1 gene product of Saccharomyces cerevisiae. Curr. Genet. 1991, 19, 333-337.

(28) Wehrs, M.; Tanjore, D.; Eng, T.; Lievense, J.; Pray, T. R.; Mukhopadhyay, A. Engineering Robust Production Microbes for Large-Scale Cultivation. Trends Microbiol. 2019, 27, 524-537.

(29) Joska, T. M.; Mashruwala, A.; Boyd, J. M.; Belden, W. J. A universal cloning method based on yeast homologous recombination that is simple, efficient, and versatile. J. Microbiol. Methods 2014, 100, $46-51$.

(30) Ruiz, E.; Talenton, V.; Dubrana, M.-P.; Guesdon, G.; LluchSenar, M.; Salin, F.; Sirand-Pugnet, P.; Arfi, Y.; Lartigue, C. CReasPyCloning: A Method for Simultaneous Cloning and Engineering of Megabase-Sized Genomes in Yeast Using the CRISPR-Cas9 System. ACS Synth. Biol. 2019, 8, 2547-2557.

(31) Gibson, D. G.; Benders, G. A.; Axelrod, K. C.; Zaveri, J.; Algire, M. A.; Moodie, M.; Montague, M. G.; Venter, J. C.; Smith, H. O.; Hutchison, C. A. One-step assembly in yeast of 25 overlapping DNA fragments to form a complete synthetic Mycoplasma genitalium genome. Proc. Natl. Acad. Sci. U. S. A. 2008, 105, 20404-20409.

(32) Gibson, D. G.; Benders, G. A.; Andrews-Pfannkoch, C.; Denisova, E. A.; Baden-Tillson, H.; Zaveri, J.; Stockwell, T. B.; Brownley, A.; Thomas, D. W.; Algire, M. A.; Merryman, C.; Young, L.; Noskov, V. N.; Glass, J. I.; Venter, J. C.; Hutchison, C. A.; Smith, H. O. Complete Chemical Synthesis, Assembly, and Cloning of a Mycoplasma genitalium Genome. Science 2008, 319, 1215.

(33) Akada, R.; Hirosawa, I.; Kawahata, M.; Hoshida, H.; Nishizawa, Y. Sets of integrating plasmids and gene disruption cassettes containing improved counter-selection markers designed for repeated use in budding yeast. Yeast 2002, 19, 393-402.

(34) Olesen, K.; Franke Johannesen, P.; Hoffmann, L.; Bech Sorensen, S.; Gjermansen, C.; Hansen, J. The pYC plasmids, a series of cassette-based yeast plasmid vectors providing means of counterselection. Yeast 2000, 16, 1035-1043.

(35) Mumberg, D.; Muller, R.; Funk, M. Yeast vectors for the controlled expression of heterologous proteins in different genetic backgrounds. Gene 1995, 156, 119-122.

(36) Bitinaite, J.; Rubino, M.; Varma, K. H.; Schildkraut, I.; Vaisvila, R.; Vaiskunaite, R. USER friendly DNA engineering and cloning method by uracil excision. Nucleic Acids Res. 2007, 35, 1992-2002.

(37) Lee, S. W.; Oh, M. K. A synthetic suicide riboswitch for the high-throughput screening of metabolite production in Saccharomyces cerevisiae. Metab. Eng. 2015, 28, 143-150.

(38) Lõoke, M.; Kristjuhan, K.; Kristjuhan, A. Extraction of genomic DNA from yeasts for PCR-based applications. BioTechniques 2011, $50,325-328$ 